\title{
ALCALÁ DE GUADAIRA EN EL SIGLO XIII Conquista y repoblación
}

\author{
MANUEL GONZÁLEZ JIMÉNEZ \\ Universidad de Sevilla
}

\section{LA CONQUISTA DE ALCALÁ DE GUADAIRA}

La conquista de Andalucía por Fernando III se desarrolla a lo largo del segundo cuarto del siglo XIII, entre 1225 y 1248, año este en que se procede a la ocupación de Sevilla, capital de los territorios peninsulares controlados por los almohades. Tras unos tímidos tanteos y la conquista de importantes enclaves en el Alto Guadalquivir (Andújar, Baeza, Quesada y Úbeda, entre otras plazas), en 1236 tiene lugar la conquista de Córdoba, cuya repoblación se ultima entre 1240-1241. A partir de este momento los acontecimientos se precipitaron, sin duda porque, tras la desaparición del caudillo murciano lbn Hud, asesinado en Almería en 1237, y la crisis final del Imperio almohade, ningún obstáculo serio se oponia a los avances de los ejércitos castellanos. El único obstáculo podía haber sido el rey de Granada Muhammad Ibn Alhmar. Pero la pérdida de Arjona, su ciudad natal, en 1244, habia puesto de manifiesto su incapacidad para enfrentarse con posibilidades de éxito al rey de Castilla. En 1246 entregaba Jaén a Fernando III, declarándose además vasallo suyo. Granada quedaba asi al margen de la contienda (1).

Las operaciones preparatorias del cerco de Sevilla debieron iniciarse en el verano de 1246, aunque la Primera Crónica General, fuente de información casi única para todo lo que se refiere a los aspectos militares de los años finales del reinado de Fernando III, parece fechar tales acontecimientos en 1247. En efecto, en el cap. 1071, se afirma que el rey, tras la conquista de Jaén, permaneció en la ciudad durante ocho meses (2). Si esto fue así, la ocupación de Alcalá de Guadaira habría que fecharla en 1247 y no en 1246 como parece lo más probable y lógico (3). 
La conquista de Alcalá fue resultado de una operación de tanteo contra Sevilla organizada por el rey apenas concluido el asedio de Jaén y firmado el pacto de vasallaje del rey de Granada (4). La Crónica refiere la operación como si de una expedición de tanteo se tratara. En efecto, las tropas del rey, a quien acompañaban su hermano el infante don Alfonso de Molina, su hijo el infante don Enrique y los maestres de las Órdenes de Calatrava y Santiago, «non passauan por trezientos caualleros arriba», a las que habría que añadir la caballeria y el peonaje del concejo de Córdoba. Un ejército muy menguado, en el cualquier caso, como para acometer la empresa del cerco de Sevilla (5). Ello explica, sin duda, que el rey se limitase a saquear las tierras de Carmona (6) y a tantear las defensas de Alcalá de Guadaira, cuyos habitantes, temerosos de los efectos de una resistencia imposible, se apresuraron a pedir del rey una honrosa capitulación. Por otra parte, la presencia en la hueste de Fernando III de un importante contingente de caballería mandado por el propio rey de Granada, que cumplía así su deber de vasallo del rey castellano, facilitó las cosas. Los de Alcalá se entregaron al rey granadino, y éste a su vez entregó la villa y la fortaleza a Fernando III (7). Era, si hemos de aceptar la fecha tradicional, el día 21 de septiembre de 1246, festividad de San Mateos. Según el analista sevillano Ortiz de Zúñiga, el rey dejó por frontero en Alcalá al noble castellano don Rodrigo Álvarez, al frente de la guarnición que se hizo cargo de la importante fortaleza de Alcalá (8)

\section{SITUACIÓN DE LOS MUDÉJARES}

No se nos ha conservado el texto de la capitulación otorgada por Fernando III a los moros de Alcalá en 1246. Pero, de todas formas, es seguro que tal acuerdo existió, ya que a él se alude en el privilegio en virtud del cual Alfonso $X$ concedió el señorío de la villa al cabildo de la Catedral hispalense. En sus líneas básicas debía ser semejante a otros acuerdos de esta índole concedidos por el mismo rey en años anteriores, y cuyos textos también han desaparecido.

Pero manejando toda la información dispersa que sobre los mudéjares andaluces nos ofrece la documentación de tiempos de Alfonso $X$, he podido reconstruir buena parte de los aspectos generales que debian contemplar las capitulaciones otorgadas por el rey a los moros. Los más importantes serían los siguientes:

$\left.1 .^{\circ}\right)$ Mantenimiento de su ley y de sus «fueros.

$\left.2 .^{\circ}\right)$ Permanencia de la estructura tradicional de la aljama o comunidad, presidida por su alcalde o alcayad, en la que se integraban especialmente los viejos y personas de más prestigio de la localidad. 
$\left.3 .^{\circ}\right)$ Mantenimiento también del mismo régimen fiscal que tenian en época almohade.

$\left.4 .^{\circ}\right)$ Respeto a sus costumbres y modo de vida tradicional, como se comprueba en la autorización de tener baños, tiendas, molinos y alhóndigas «a la costumbre de los moros", como se lee en un acuerdo con los moros de Morón hecho en tiempos de Alfonso $X$.

$\left.5 .^{\circ}\right)$ Por último, derecho a marchar libremente a donde quisieran.

Al amparo de estas garantías, buena parte de la población musulmana de Alcalá, debió permanecer en la villa gobernada por su alcalde o alcayad Hamet Abén Paxat.

De todos estos aspectos, el mejor documentado es el que se refiere a la situación fiscal de los mudéjares, que, en esencia, reproducía el esquema tradicional, que consistía en el pago al rey del diezmo de todas las cosechas; un impuesto de capitulación, llamado genéricamente en los documentos «el pecho de los moros» o alfitra, y un impuesto sobre la tierra conocido con el nombre de almarjal. Es posible que también pagasen un impuesto sobre el ganado, llamado en la documentación valenciana de la época azaque, y que en algunos casos los moros estuvieron sometidos a prestar ciertos servicios personales para la reparación de las fortalezas o para el mantenimiento de edificios de cierta importancia, como sucedía en Córdoba, donde los carpinteros, serradores y albañiles moros debían trabajar gratis dos días al año en las obras de conservación de la mezquita catedral (9).

El régimen fiscal de los mudéjares - aunque se presente como prolongación del anterior - distaba mucho de ser generoso, y es probable que los castellanos se encargasen de endurecerlo aún más en los años que siguieron a la conquista. Ello explicaría, entre otras, la sublevación casi general de los mudéjares andaluces y murcianos en la primavera de 1264 y la emigración de muchos de ellos a Granada, antes incluso de producirse la revuelta (10).

\section{PRIMERA REPOBLACIÓN DE ALCALÁ}

Hasta 1253, fecha del repartimiento de Sevilla, Alcalá de Guadaira no fue sino una pieza importante del sistema militar recién consolidado por la conquista de la ciudad. En consecuencia, la población cristiana debía limitarse al reducido contingente de soldados acantonados en su fortaleza. Es posible, que por estas fechas — rompiendo de alguna forma lo pactado en 1246la población mudéjar hubiese sido ya desplazada de la villa al Arrabal, y que 
sus casas comenzasen a ser ocupadas por un primer grupo de repobladores de los que casi nada sabemos.

De todas formas, parece que esta primera implantación de pobladores cristianos en la Villa, de la que ningún vestigio claro nos ha llegado, debió ser de escasa importancia, o todo lo más, acabaría integrándose en el grupo de soldados/repobladores que pudieron asentarse en Alcalá tras haber recibido tierras en su término y casas en la villa como pago a los servicios prestados en la conquista de Sevilla y/o, como parece probable, por su condición de repobladores.

Este grupo de soldados/repobladores recibió casas en Alcalá y heredades en la aldea o hacienda de Borgalhamar, que el rey habia reservado inicialmente para sus galeras. Los beneficiarios pertenecian a cinco cuadrillas de almogavares o peones, a las órdenes de sus respectivos jefes de grupo o almocadenes. A cada uno de ellos se entregaron lotes de tierra, de acuerdo con su categoría militar. $Y$, así cada uno de los cinco almocadenes recibió un lote consistente en dos yugadas de tierra de labor (unas 60 has.), dos aranzadas de viña; tres aranzadas de olivar, y una aranzada y media de huerta; mientras que cada uno de los almogavares - 57 en total- recibió una yugada de tierra de labor; dos aranzadas de viña; dos de olivar y una de huerta. Así pues, el total de bienes repartido ascendió a 60 casas, 61 yugadas de tierra de labor (unas 1.830 has.), 117 aranzadas de olivar, otras tantas de viña y unas 58 y media de huerta.

De ser correcta la suposición de que los beneficiarios de este reparto-que figura en el repartimiento de Sevilla, al final de los donadíos menoresestaríamos ante la nómina más antigua y autorizada de repobladores cristianos de Alcalá de Guadaira (11).

Pero los repartos efectuados en término de Alcalá no beneficiaron sólo a repobladores de la villa. La gran extensión del antiguo término de Alcalá, que se prolongaba hasta el Guadalquivir, englobando el de Dos Hermanas, y lo exiguo del poblamiento cristiano de la villa, asentado a la sombra del castillo, explican las numerosas donaciones hechas por Alfonso $X$ que registra el libro del repartimiento de Sevilla.

Los lugares y fincas que figuran en dicho texto pueden agruparse, en razón de sus beneficiarios, en las siguientes categorías:

1) Lugares y fincas entregados en bloque o en parte a beneficiarios de donadios mayores (miembros de la familia real, alta nobleza y Órdenes Militares): 
- BORGABENALCADI, dada al infante don Enrique, hermano de Alfonso $X$.

- BORGABENAUN, donde la Orden de San Juan recibió undonadío de diez yugadas de tierra de labor (unas 300 has.)

- BORGABENHALDON, donde la Orden de Uclés recibió, junto con la torre de la finca, 30 yugadas de tierra de labor (unas 900 ha.)

- BORJASANTAREM, concedida íntegramente a don Ruy López de Mendoza, almirante de Castilla.

- CERRAJAS, dada a don Rodrigo Gómez de Galicia (12).

- FOXAT, donadío de don Rodrigo Álvarez, primer alcalde cristiano de Alcalá.

- MACHAR AZOHIRI, entregado a don Fernando, hijo del antiguo rey moro de Baeza.

- VILLANOVA AZEQUILLA, donadío de don Gutiérrez Suárez de Meneses.

- ZAHELA, la mitad de la cual, con un cortijo y una torre, fue entregada a don Rodrigo Álvarez, ya citado.

- GANDUL y MARCHENILLA, donde recibió tierras de labor el mentado don Rodrigo Álvarez.

2) Alcarias donde recibieron tierras beneficiarios de donadios menores:

MACHAR ABENHAQUIM, cortijo atribuido inicialmente a las galeras del rey y entregado a 28 hombres de las "Compannas» del infante don Enrique.

3) Tierras reservadas por el rey para su almacén:

El rey se reservó como propias, en diversos sitios, unas 1.050 aranzadas de olivar y unas 94 yugadas de tierra de labor (unas 2.820 ha.) ubicadas en Bulbal, Tavilla, Facaxtalmi, Bibares, Arenales y Carchena (13).

4) Aldeas reservadas para las galeras del rey:

De las tres que inicialmente se reservó el rey para este fin sólo retuvo la de BORGABENDARIZ (14).

5) Por último, una larga serie de cortijos y alcarias entregado al concejo de Sevilla para heredar en ellos a los pobladores de la ciudad: 
- ALCARIAS: ALJUBET, BORGABENHALDON, BORGABENÇOMA, MACHAR ABDILQUIVIR, MACHAR AXARAFI, MACHAR YANCO, MALIX, QUINTOS, QUARTOS, TOCORICOBIZ Y ZAHELA.

\section{- CORTIJOS. CORTIGENA y LA MEMBRILLA (15).}

En conjunto las tierras entregadas ya sea a beneficiarios de donadíos 0 a pobladores, ascendieron a una 900 yugadas de tierra de labor (unas 27.000 ha.) y a unas 20.000 aranzadas de olivar e higueral, cultivos absolutamente predominantes en el término de Alcalá de Guadaira, especialmente en su sector norte. Algunas alcarias, como las de Borgabendariz, Borgabenalcadí, Borgabençoma y, en especial, la de Abenhaquín estaban dedicadas casi por completo al cultivo de la higuera. De todas formas, el olivar aparece en el texto como el cultivo rey, si bien el arbolado de algunas fincas, como la de Quartos, debió quedar, tras el asedio de Sevilla y por su proximidad al campamento cristiano, literalmente devastado. Sólo así se justifica que en una superficie de 1.160 aranzadas quedasen tan sólo 3.000 pies de olivar o lo que es lo mismo, a razón de 50 pies por aranzada, una superficie en explotación de nada más que 60 aranzadas.

Entre los bienes repartidos en término de Alcalá ocupan un lugar preferente los molinos harineros ubicados en las márgenes del río Guadalquivir o junto a los «caños» que conducían el agua a Sevilla. Algunos de ellos están perfectamente documentados, como el de Abén Haroça, entregado por el rey a don Pedro Pérez, canciller de doña Juana de Ponthieu, viuda de Fernando III (16); o el molino de Abén Ocba, que perteneció a Hamet Abén Paxat, el alcalde de los moros que permanecieron en Alcalá de Guadaira tras la conquista (17); o los nueve o más molinos otorgados por Alfonso $X$ al concejo de Sevilla, "que son en la azequia de la montanna de Alcalá de Guadayra” (18); o, finalmente, los molinos concedidos por Fernando III a la Orden de Alcántara, «los que son más çerca de la puente por ó passé yo con mi hueste quando vine de Alcalá sobre Sevilla» (19). También los había en Cerrajas, y de gran valor, ya que la Orden de Calatrava los retuvo cuando cedió la propiedad de la alcaria citada(20).

\section{ALCALÁ BAJO LA JURISDICCIÓN DE SEVILLA}

La presencia masiva en término de Alcalá de propiedades de vecinos de Sevilla 0 , al menos, atribuidas al concejo hispalense para ser repartidas entre pobladores de la ciudad, se explica sólo por la vinculación jurisdiccional de la villa con respecto a la urbe. Todavía no se habia acuñado la expresión que definía a Alcalá como "guarda e collación de Sevilla»; pero, induda- 
blemente, la villa cumplía ya algunas de estas funciones. Desde luego, desde el punto de vista militar, su castillo era para la ciudad una garantía de seguridad absolutamente irreemplazable. Pero, además, Alcalá estaba íntimamente asociada al abastecimiento de la ciudad: el agua, los molinos y el mismo pan de Alcalá significaban mucho para Sevilla. Sólo así se entiende que, desde el primer momento, Alcalá aparezca tan estrechamente asociada a Sevilla y que por eso mismo el rey la sometiese a su jurisdicción.

Efectivamente, en diciembre de 1253, concluido ya el repartimiento de Sevilla, Alfonso $X$ concedió términos propios a la ciudad, al tiempo que le confirmaba el fuero otorgado por Fernando III años antes. Pues bien, entre las villas incorporadas a la jurisdicción y fuero de Sevilla figura Alcalá de Guadaira.

En este mismo privilegio el rey se reservó las rentas de los almojarifazgos o aduanas de Tejada, Sanlúcar la Mayor, Aznalcázar, Constantina y Alcalá de Guadaira, villas todas ellas dependientes de la ciudad, y vinculó de tal manera a sus pobladores con la ciudad que en la práctica les otorgó la condición de vecinos de la propia Sevilla. El texto es de un gran interés y dice como sigue:

«Et tengo pora mí e pora todos aquellos que regnaren después de mí en Castiella e en León las rentas de los almoxarifadgos con sus pedidos de taxada, de Solúcar la Mayor, e de Heznalcáçar e de Aicalá de Guadayra e de Costantina, en tal manera que todos los christianos que son hy oy pobladores e serán daquí adelante pora siempre iamás en estos cinco logares sobredichos, que fagan con el conceio de Seuilla todos aquellos fueros e todos aquellos derechos que fazen e que farán los que son e que serán pobladores e moradores en Seuilla e en sus términos, e que ayan esse fuero mismo" (21).

En cuanto villa dependiente de Sevilla, Alcalá recibió el fuero de la ciudad, por el que continuó rigiéndose en adelante, aún en los momentos en que dejó de depender de la jurisdicción sevillana.

\section{ALCALÁ, SEÑORIOO DE LA IGLESIA DE SEVILLA}

En septiembre de 1258 Alfonso X entregó a la Iglesia Hispalense «la villa et castiello» de Alcalá de Guadaira, a la que segregó de la jurisdicción del concejo sevillano. Este importante acontecimiento hay que interpretarlo dentro de las medidas adoptadas por el monarca para dotar económicamente a la Iglesia de Sevilla (22). Ésta era una tarea absolutamente inaplazable ya 
que, a pesar de que hacia ya diez años que se había producido la conquista de la ciudad, la sede arzobispal y el cabildo catedralicio hispalenses no habian recibido los bienes que correspondian a su rango. Por razones que desconocemos, Alfonso $X$ dejó pasar la oportunidad que le ofreció el repartimiento de Sevilla, en el que la Iglesia hispalense recibió muy pocos bienes: algunas rentas de dinero, otorgadas ya por Fernando III, y la propiedad de todas las mezquitas de la ciudad, y poco más.

Hasta finales de 1257 el infante don Felipe retuvo la dignidad y las rentas de la mitra hispalense, de la que era arzobispo electo a pesar de haber contraido matrimonio en 1252 con la princesa doña Cristiana de Noruega. En 1258 la sede arzobispal sevillana aparece en la documentación real como «vaga» o vacante, aunque, sin duda, el rey hacía tiempo que tenía decidida la designación de don Remondo, obispo de Segovia. En realidad el obispo segoviano, hombre de confianza el rey, hasta el punto de haber organizado las operaciones del repartimiento de Sevilla en 1253, venía administrando la Iglesia hispalense desde hacía años. Y a instancias suyas debió iniciarse en 1258 el proceso de dotación de la Iglesia sevillana, precisamente con la concesión de Alcalá de Guadaira al cabildo de la catedral. A partir de este momento y hasta 1279, Alfonso $X$ cumplió con generosidad esta tarea ineludible. $Y$, asi, el arzobispo y el cabildo hispalense se convirtieron en pocos años en señores de Alcalá de Guadaira y Constantina (1258); Cazalla, Brenes, Tercia, Umbrete y Solúcar de Albaida (1260); Gelves (1272); Gelo (1274); Cambullón, Torre del Alpechin y Las Chozas (1277); Almochachar y Rianzuela (1278), y, finalmente, Almonaster y Zalamea (1279).

Con la excepción de las dos primeras donaciones -Alcalá y Constantina, dos villas pertenecientes inicialmente a la jurisdicción de Sevilla-, el resto eran aldeas o alcarias de poca importancia demográfica. Cazalla (hoy Puebla de Cazalla) debia tener muy poca población, aunque era villa de un cierto valor estratégico. Pero la más sorprendente de todas estas donaciones era la de Alcalá. Y es que resulta extraño que el rey desvinculase de Sevilla una localidad tan próxima a ella y de la importancia estratégica y económica de Alcalá. Por otra parte, la defensa de su castillo, el pago de los salarios de una guarnición permanente y la reparación de su fábrica eran algo que desbordaba la capacidad económica de a Iglesia sevillana. La única explicación plausible que se me ocurre para este hecho es suponer que Alfonso X estaba convencido de que ya no eran necesarias tantas precauciones defensivas, ya que Alcalá habia perdido su significación estratégica. Si éste fue el pensamiento del rey, los acontecimientos posteriores - la revuelta mudéjar de 1264, la guerra con Granada y la intervención en la misma de los benimerines-- se encargarían de demostrarle dramáticamente que no estaba en lo cierto. 
La concesión de Alcalá a la Iglesia sevillana se nos ha conservado en un precioso privilegio rodado, fechado a 13 de septiembre de 1258, que se guarda en el Archivo de la Catedral de Sevilla. En sus primeras líneas el rey justifica la entrega de la villa y del castillo de Alcalá

por grand sabor que auemos de heredar e de fazer bien e onrra alla (sic) eglesia cathedral de Sancta Maria de la noble çibdad de SeuiIla, la que ganó e fundó el muy noble e much onrrado, el rey don Ferrando, nuestro padre, e nos con él, e le ganamos de moros e la poblamos de cristianos a seruiçio de Dios de de la Virgen Sancta María, e de la Eglesia de Roma...»

El privilegio reproduce las cláusulas habituales en documentos de esta indole. El rey entrega la plena posesión de la villa — «con todas sus entradas e con todas sus salidas... con montes, con ríos, con pastos e con todos sus términos poblados e por poblar...»-, con sus rentas y derechos al cabildo de la Catedral de Sevilla. Como era norma, el rey se reserva el impuesto de la moneda forera, la propiedad de las minas que pudieran descubrirse en el término y el señorío eminente de la Corona en cuestiones de ámbito supralocal - "que faga por nos guerra e pas»- y generales ("que entre hy nuestro Adelantado, assi commo entra en todas las otras villas de la Frontera que son de las Ordenes e de les eglesias cathedrales»).

El rey obliga a los nuevos señores a respetar las propiedades o heredamientos concedidos en Alcalá tanto por su padre como por él mismo. $Y$, al mismo tiempo, mantiene a Alcalá dentro del término o tierra de Sevilla, de forma que los vecinos de ésta y de su tierra, y los de Alcalá pudiesen seguir gozando de comunidad de pastos y aguas en sus términos respectivos. El mantenimiento de la vinculación con la ciudad se extiende también a los aspectos legales y jurídicos, aún reconociendo al cabildo de la Catedral la capacidad de nombrar en Alcalá alcaldes y alguaciles. Pero, en los demás Alcalá de Guadaira siguió rigiéndose por «el fuero de Seuilla, onde an el Fuero de los Juyzios» o Fuero Juzgo, estableciendo el rey como jueces de apelación o de alzada a los alcaldes de Sevilla.

Finalmente, Alfonso X obligó al cabildo de la catedral hispalense a respetar a los moros que permanecian en la villa las capitulaciones y «pleytos que an connusco, assí como dizen las cartas que de nos tienen».

Con estas salvedades, el rey reconoce al cabildo plena autoridad en la villa y absoluta capacidad para instalar pobladores cristianos y recibir de ellos los impuestos que estableciesen, asi como los otros derechos que correspon- 
dían anteriormente al rey, dejando naturalmente a salvo el señorio eminente de éste, expresado en las limitaciones mismas de la concesión.

\section{SEGUNDA REPOBLACIÓN DE ALCALÁ}

No se sabe con exactitud cuándo dejó de pertenecer Alcalá de Guadaira al señorío de la catedral de Sevilla. Es probable que Alfonso $X$ recuperase para el realengo la villa y el castillo a raíz de las incursiones benimerines iniciadas en 1275 (23). Podriamos, incluso, suponer que fue el propio cabildo catedralicio quien renunció a la responsabilidad de mantener en tan difíciles circunstancias la defensa del alcázar alcalareño. En cualquier caso la devolución se hizo, que sepamos, sin compensaciones de ningún tipo. Ello explicaria la ausencia de documentos que permitan fechar el acontecimiento. Desde luego, en 1279-80 la defensa de Alcalá y de su castillo estaba a cargo del concejo de Sevilla, según veremos más adelante.

Las razzias de los benimerines, que se prolongaron en la zona sevillana hasta finales del verano de 1277 , debieron afectar gravemente al poblamiento de Alcalá de Guadaira. Sabemos, en efecto, que los alrededores de Sevilla y la comarca del Aljarafe fueron saqueados ferozmente en agosto de 1277. El cronista Ibn Abi Zar afirma que los marroquies tomaron al asalto los castillos de Cantillana, Guillena y Alcolea, destruyendo sus fortificaciones, y que incendiaron las casas y castillos del Aljarafe (24). Parece probable que, tras estas incursiones, la población de Alcalá quedase reducida casi a la guarnición que defendía su castillo.

Así las cosas, a fines de 1277 se produjo la intervención personal de Alfonso $X$ para remediar el estado de despoblación y abandono a que había llegado Alcalá de Guadaira. Para restaurar la villa, el rey ordenó una nueva repoblación de Alcalá. La operación formaba parte de otra más compleja tendente a reestructurar el sector fronterizo sevillano con el reino de Granada, el más frágil de toda la frontera. Años antes, a raíz de la revuelta mudéjar, Alfonso $X$ había decidido dar mayor participación a las Órdenes Militares en la defensa de la línea fronteriza. De esta forma, en 1264, la Orden de Calatrava recibió Osuna, con el encargo de trasladar a esta villa el "convento mayor» de la Orden que estaba, como es sabido, en Calatrava la Nueva. Tres años más tarde, la Orden de Santiago recibió la importante plaza de Estepa. Ahora, tras el fracaso de Alfonso $X$ ante los muros de Algeciras y la amenaza de nuevas incursiones de los benimerines, habia que completar la militarización de la frontera. Fue así como la Orden de Alcántara recibió el encargo de defender los castillos de Morón y Cote, y la de Calatrava, el de Cazalla. Sólo 
faltaba reforzar la posición de Alcalá de Guadaira, verdadero antemural de Sevilla y punto hacia el que convergían todos los caminos de penetración desde la frontera.

Desde esta perspectiva podemos entender sin dificultades la compleja operación a tres bandas emprendida por el rey en diciembre de 1279. El 15 de dicho mes la Orden de Calatrava recibía la villa y castillo de Cazalla, que pertenecia a la Iglesia de Sevilla desde 1260, a cambio del lugar de Cerraja y los demás heredamientos que la Orden poseia en término de Alcalá de Guadaira, con la excepción de los molinos. Al día siguiente Alfonso $X$ otorgaba al cabildo de la catedral de Sevilla Almonaster y Zalamea a cambio de CazaIla. $Y$ el 11 de enero de 1280 el rey aprobaba el trueque efectuado entre el cabildo de la catedral y el concejo de Sevilla el día 4 del mismo mes, en virtud del cual aquél entregaba a la Orden de Calatrava Cazalla en nombre del concejo hispalense, y recibía de éste a cambio Almonaster y Zalamea (25).

En apariencia, el concejo de Sevilla obtuvo una pobre compensación: Cerrajas a cambio de Almonaster y Zalamea. Pero, sin duda, la operación que hemos descrito significaba más de lo que la documentación indica. De entrada, con la disponibilidad de Cerrajes se conseguian más tierras para repartir entre repobladores y, de paso, se alejaba de Sevilla la posibilidad de que la Orden de Calatrava pudiese crear en torno a la finca un núcleo de población separado de la jurisdicción sevillana. Pero, además, mediante la instalación de Alcalá de un grupo reaimente importante de pobladores que asumiesen la tarea de vigilar la villa y defender su castillo. Sevilla se liberaba de la pesada carga que ello suponía para las arcas municipales (26). Y, por último, con la cesión al cabildo de la catedral de unos lugares hasta entonces dependientes de la jurisdicción sevillana se cerraba el paso a cualquier reclamación que en el futuro la Iglesia hispalense pudiese efectuar sobre Alcalá de Guadaira.

\section{LA CARTA-PUEBLA DE ALCALÁ}

La atracción de pobladores emprendida por Alfonso $X$ a finales de 1279 culminaría en mayo del siguiente año con la concesión a los pobladores de Alcalá de Guadaira de una carta-puebla. Este precioso documento ha desaparecido en su versión original y desconozco si en el Archivo Municipal de la villa o en alguna otra parte existe una copia del mismo. Por fortuna, el P. Leandro Flores publicó un amplio extracto del privilegio real, copiándolo de un Inventario de Papeles del Cabildo que se conservaba en el Archivo alcareño a comienzos del siglo pasado (27). 
La carta-puebla de Alcalá reviste la forma de un contrato entre el rey y los 150 pobladores que se pretendía instalar en la villa. Se trata, pues, de un típico contrato «ad populandum", redactado en forma de privilegio real, donde se consigna, por un lado, una serie de concesiones y exenciones hechas por el monarca a los pobladores, $y$, por otro, las obligaciones que en contrapartida asumen los que estaban dispuestos a instalarse en la localidad.

El primer dato de interés que nos ofrece el documento es el número de pobladores previstos para Alcalá: 150, cifra que, dadas las circunstancias, habría que conisiderar como alta si la comparamos con los contingentes de pobladores asentados en otras localidades repobladas a mediados o a finales del siglo XIII (28).

El conjunto de pobladores recibió una serie de heredamientos, adquiridos expresamente para ser repartidos entre los nuevos repobladores: Cerrajas y, tal vez, las heredades que habian sido de Rodrigo Esteban, alcalde de Sevilla, Diego Alfonso, alcalde de Córdoba, y Nuño Fernández; o cedidas por el concejo de Sevilla, como la alcaria de Zahela, que había correspondido en parte al heredamiento de la ciudad en el repartimiento de 1253 (29); o, finalmente, propiedades que, por haber sido abandonadas por sus primeros titulares o por cualquier otra causa, pertenecian a la propia corona. La cartapuebla consigna con exactitud en casi todos los casos la ubicación, extensión y nombres de los anteriores propietarios de las tierras entregadas a los repobladores de Alcalá, tal como se refleja en el cuadro siguiente:

\begin{tabular}{|c|c|c|c|}
\hline ALCARIA & ANTERIOR PROPIETARIO & TIERRA DE LABOR * & OLIVAR * \\
\hline \multirow[t]{2}{*}{ ZAHELA } & Concejo de Sevilla & - & 一 \\
\hline & Don Rodrigo Álvarez & - & 830 \\
\hline FOXAT & Don Rodrigo Álvarez & - & 1.200 \\
\hline CERRAJAS & Orden de Calatrava & 20 & 1.000 \\
\hline BENABORRA & Orden de Calatrava y de & 16 & - \\
\hline ONRRATA & Santiago & 6 & - \\
\hline \multirow[t]{2}{*}{ LERENA } & Antón de Farias & 4 & - \\
\hline & Per lbáñez de la Calzada & 3 & - \\
\hline \multirow[t]{3}{*}{ BENAGIRA } & Hijo de Pedro de la Calzada & 3 & - \\
\hline & Esteban de Ferrera & 6 & - \\
\hline & Gutierre Pérez & 6 & - \\
\hline \multirow[t]{2}{*}{ CORTIGENA } & Hijos de Martín Meléndez & 12 & - \\
\hline & TOTAL $\ldots \ldots \ldots \ldots \ldots \ldots \ldots \ldots \ldots \ldots \ldots \ldots \ldots$ & 79 & 2.030 \\
\hline
\end{tabular}


Algunas de las personas reseñadas figuran en la documentación, como el alcaide don Rodrigo Álvarez, que había recibido en el repartimiento de Sevilla la alcaria de Foxat o Frostad, la mitad de la de Zahela, con 300 aranzadas de olivar, un cortijo y una torre, y 220 yugadas de tierra de labor entre Gandul y Marchenilla. Esteban de Ferrera y Martín Meléndez fueron caballeros hidalgos heredados en Sevilla con los 200 caballeros de linaje establecidos por Alfonso $X$ en la ciudad (30). Por desgracia el documento extractado por el $P$. Flores no permite valorar la entidad de las propiedades de olivar, higueral, tierras de pan y huertas que poseian en Alcalá los alcaldes de Sevilla y Córdoba citados en el texto, y Nuño Fernández, que bien pudiera ser uno de los varios caballeros hidalgos que figuran con este nombre en el libro del repartamiento de Sevilla (31).

Además de estas heredades, Alfonso X otorgó a los repobladores de Alcalá exención total de pechos, pedidos y préstamos, hospedaje y facendera, como era normal en muchas localidades de frontera.

En contrapartida, los repobladores se comprometían individualmente a participar en las velas y atalayas del castillo y en la vigilancia del Arrabal, morando «en el castillo con vuestros cuerpos». Más aún, para garantizar tanto la repoblación de la villa como su defensa, los repobladores se comprometieron a no disponer de los heredamientos recibidos hasta pasados seis años. La única excepción que contempla el privilegio alfonsí es en el caso de que el titular del heredamiento cayese cautivo de moros. En tal supuesto, podía disponer de sus bienes, pero siempre que el comprador se comprometiese a hacer vecindad en la villa, cumpliendo de esta forma los servicios y prestaciones militares a los que estaba obligado el vendedor. 


\section{NOTAS}

(1) Sobre la conquista de Andalucía ver J. GONZÁLEZ, "Las conquistas de Fernando III en Andalucia», Hispania, 25 (1948).

(2) Primera Crónica General de España; ed. R. MENÉNDEZ PIDAL, Madrid, 1955, cap. 1071, p. 747. "Ocho meses moro y el rey don Fernando en Jahen desque la aouo ganada..."

(3) Todo hace suponer que la conquista de Alcalá se afectuó en 1246. Y, aunque la Crónica General fecha los acontecimientos en 1247, sabemos que cuando se inició el cerco de Sevilla en el verano de dicho año, el rey salió con sus huestes desde Alcalá, lo que indica que ya estaba conquistada.

(4) Jaén debió conquistarse a fines de marzo o a comienzos de abril de 1246. Un documento de 13 de abril aparece fechado "Eo videlicet anno que rex Granate factus est vassallus regis Castelle et osculatus est manu eius, et in signum domini Jahenni tradidit illis. Archivo Histórico Nacional, Ordenes Militares, Santiago, Alanje, cajón 52, n. 7.

(5) Primera Crónica General, cap. 1072, pp. 747-48.

(6) Ibid., p. 748.

(7) Ibid., p. 748, «Et estando alli el rey don Fernando en Carmona, veno y a el rey de Granada, su vasallo, con quinientos caualleros, quel venia a seruir. Et desque el rey don Fernando ouo talado et astragado a Carmona, mouio ende su hueste et fuese para Alcala de Guadaira; et los moros de Alcala, quando sopieron que el rey de Granada yua y, salieron et dieronse a el, et el dio luego el castiello a su sennor el rey don Fernando".

(8) Diego ORTIZ DE ZUÑIGA, Anales eclesiásticos y seculares de Sevilla, Madrid, 1795, año 1247,8 .

(9) Tomo estos datos de mi ponencia presentada al $V$ Coloquio de Historia Medieval Andaluza (Córdoba, noviembre, 1986) en prensa.

(10) En 1253 el alcalde Abén Paxat había ya abandonado Alcalá de Guadaira, según consta en un documento en el que se alude a un molino «que tue de Hamet auén Paxat, alcayat que fue de los moros que fincaron en Alcalá de Guadayra”. Edito el doc. en Diplomatario Andaluz de Alfonso $X$ (en prensa), n. 96.

(11) Cfr. J. GONZÁLEZ, Repartimiento de Sevilla (2 vols.), Madrid, 1951, Il, pp. 107-109 Doy el texto integro de este reparto en Apéndice I.

(12) En 1256 Alfonso $X$ autorizó a don Rodrigo Gómez de Galicia a ceder a la Orden de Calatrava la alquería de Cerrajas, permaneciendo esta propiedad en poder de la Orden hasta 1279. Diplomatario Andaluz de Alfonso X, n. 170

(13) J. GONZÁLEZ, ob. cit, II. p.

(14) De todas formas, el rey entregó 175 ars. de olivar a cinco beneficiaros $=100$ ars. a Pedro González de Quesada y a su hermano Gutierre González; 30 ars. a Martín Muñoz; otras 
30 a Arias Pérez, botero, y 15 ars. a Juan Díaz, caballerizo del rey. Cfr. J. GONZÁLEZ, ob. cit., il, p. 155.

(15) En realidad el concejo de Sevilla recibió algunas alcarias más en el término de Alcalá. Se trata de lugares yermos que, en consecuencia, no fueron valorados por los repartidores. Entre ellos figuraban Giçirat Fisicar (J. GONZÁLEZ, ob. cit. II, p. 116) o Gezira Faxequit, según doc. de Alfonso $X$ de diciembre de 1253, que edito en Diplomatario..., n. 99 y Machar Abnalget (J. GONZÁLEZ, ibid.)

(16) Diplomatario..., n. 91. Estos molinos pasarían por donación de su primer titular a la reina doña Juana, quienes los legó a su hijo el infante don Luis. Éste, a su vez, los daria en 1262 a Garcia Martínez. Diplomatario..., n. 260.

(17) El primer propietario cristiano de estos molinos fue Garci Martínez, ayo de la infanta doña Leonor. Lindaban con los molinos de Abén Haro'a y con los de Luet y Alcaxur, "que muele de una fuente». Diplomatario, n. 96.

(18) Diplomatario, n. 121.

(19) Ibid., n. 151. El puente aquí citado podría ser el de al-Qantara Talalhuat o «Puente de las Tablas", citado en un doc. de 1263. A. BALESTEROS, Sevilla en el siglo XIII, Madrid, 1913, ก. 126.

(20) Diplomatario, nn. 448 y 454

(21) Ibid., ก. 80.

(22) Los datos que siguen proceden de mi artículo «Propiedades y:rentas territorales del cabildo de la Catedral de Sevilla a fines de la Edad Media", Cuadernos de Historia, Anexos de la revista HISPANIA, 7 (Madrd, 1977), pp. 177-178.

(23) Cfr. Ibn ABI ZAR, Rawd al-Qirtas, trad. de Ambrosio HUICI MIRANDA, vol. II, Valencia 1964 , p. 592 ss.

(24) Ibid., pp. 611-612.

(25) Todos estos documentos se editan en mi Diplomatario, nn. 448, 449 y 454 . Los dos últimos (concesión de Zalamea y Almonaster al cabildo, y aprobación del trueque de Cazalla por los dos lugares citados) fueron editados por A. BALLESTEROS, ob. citada, nn. 221 y 223.

(26) En el acuerdo entre el concejo de Sevilla y el cabildo de la catedral, de 4 de enero de 1280, aquél reconoce que cestos heredamientos (se refiere a los que la Orden de Calatrava poseía en término de Alcalá) nos dieron a nos pora poblarlos e darlos a pobladores de Alcalá de la lazería e de la coyta e de la costa pora siempre iamás de la guarda de Alcalá de Guadayra, e del destaio e de las escuchas escusannas que nos auiemos de pagar cada anno".

(27) P. Leandro José de FLORES, Memorias históricas de Alcalá de Guadaira, Alcalá de Guadaira (Excmo. Ayuntamiento), 1983, pp. 33-34. Ofrecemos nuestra versión en Apéndice II.

(28) Cfr. M. GONZÁLEZ JIMÉNEZ, En torno a los orígenes de Andalucia. La repoblación del siglo XIII, Sevilla 1980, pp. 50-53.

(29) Como indiqué más arriba, don Rodrigo Álvarez había recibido 300 ars. de olivar en Zahela.

(30) Esteban de Ferrera, Repartimiento de Sevilla, II, pp. 133 y 227. Martín Meléndez, ibid., pp. 130 y 227. En el Archivo Municipal de Sevilla, Sec. 1Ç, carpeta 1, n. 1, se conserva el privilegio de Alfonso $X$ concediendo a este caballero heredamiento en la ciudad. Edito el doc. en Diplomatario, n. 18.

(31) Repartimiento de Sevilla, II, p. 434. 


\section{APÉNDICE I}

1253

Repartimiento de la alcaria de Borgalhamar.

J. GONZÁLEZ, Repartimiento de Sevilla, II, pp. 107-109

Borgalhamar, ques de término de Alcalá de Guadaira; en que avía 3.000 pies de olivar e de figueral para 8.000 seras de figos, e por medida de tierra 1.100 arançadas.

Dióla el rey, que la tenía para sus galeas, a estos amocadenes e a estos almogávares que aquí sonescritos, en cambio del heredamiento que le/s/ diera el rey don Fernando, con su carta plomada, de Alcalá de Guadaira, desta guisa:

- al almocadén, dos yugadas de bueyes anno e vez, e dos arançadas de vinnas, e arançada e media de huerta, e tres arançadas de olivar;

- al peón una yugada de bueyes, anno e vez, e dos arançadas de vinnas, e una de verta e dos de olivar.

E todo esto montava 60 yugadas de bueyes, ann e vez, e 60 arançadas de vinnas, e 60 arançadas de vinnas, e 50 arançadas de olivar, e 60 pares de casas en Alcalá.

E dióles el rey don Alfonso esta aldea por este heredamiento a su ventura en donadío.

(1) Domingo Sancho, almocadén, es heredero con estos 15 peones:

- Juan de Bardía

- García Navarro 
- Juan Pérez de Alvires

- Pedro Gil de Segura

- Pedro de Agreda

- Martín Domínguez

- Mingo Negrillo

- Miguel García

- Juan de Agreda

- Per lbáñez de Alcaraz

- Aparicio de Alcaraz

- Don Gil de Ellón o Ello

- Juan Martínez de Segura

- Martín Rodríguez de Martín Pérez

- Mateo de Olvera

(2) Heredó el rey a Miguel Pérz de Alcaraz, almocadén, con estos peones:

- Gonzalo de Quesada

- Yagüe de Cuéllar

- Juanes de Baeza

- Juan Pérez

- Pedro García de Tordehumos

- Martín Muñoz

(3) Heredó el rey a Domingo Perez, almocadén, heredero de Domingo Blanco, con estos 6 peones:

- Domingo Martín de Molina

- Don Gil, su hermano

- Martín de Huete

- Pedro García

- Vicente Miguel

- Domingo de Alarcón

(4) Heredó a Miguel de Vios e Ibros, almocadén, con estos 13 peones:

- Gil Pulgar

- Domingo Pérez

- Domingo Ibáñez de Ávila

- Domingo Martín de Alguinlas o Alguiulas

- Domingo Malatado

- Don Pascual de Cuenca

- Domingo de Segovia

- Rodrigo de Burgos 
- Domingo Pérez

- Pedro Ruiz de Zorita

- Domingo Pascual de Brihuega

- Martín Domingo de Ávila

- Fernando de Burgos

(5) Heredó a Esteban de Uclés, almocadén, con estos 11 peones:

- Bartolomé de Montamarta

- Bon Blanco

- Juan Pérez de Soria

- Pedro Macarena

- Martín, criado del adalid

- Domingo Ovieco

- Pedro Rodríguez, ballestero

- Domingo Gonzalo

- Bivas

- Blasco Miguel

- Domingo Miguel de Madrigal 
$\therefore$ 


\section{APÉNDICE II}

1280, mayo, 31, Sevilla

Alfonso X otorga carta-puebla a los pobladores de Alcalá de Guadaira.

EDT. L. J. FLORES, Memoria Históricas de Alcalá de Guadaira, Alcalá de Guadaira 1983, pp. 33-34.

Extracto de un «Inventario de Papeles del Cabildo" que se conservaba en el Archivo Municipal de Alcalá.

Fecha la carta en Sevilla, viernes postrimero día de mayo, en era de 1318 /annos/m

Yo Millán Pérez de Aellón lo fice escribir por mandado del rey, en veinte e ocho annos que el rey sobredicho reinó. Juan Pérez».

(1) Alfonso $X$ concede a los 150 pobladores de Alcalá «todo el término de Alcalá de Guadaira», "olivares e figuerales e tierras de pan e huertas, /e/ con lo que y á e huviere Rodrigo Esteban, nuestro alcalde de Sevilla, e con lo de Diego Alfonso, alcalde de Córdoba, e con lo de Nuño Fernández».

(2) Les entrega las alcarias de Zahela y Frostad o Foxat, y «todo quanto á Cerrajes, alcaria arriba, que se tiene con lo que nos diemos a Arquier, nuestro criado, que es de alcaria ayuso».

(3) Les entrega además en Benaborra «lo que tiene la Reina, que fue de la Orden de Santiago e de Calatrava", que ascenderia a unas 16 yugadas de tierra.

(4) En Onrrata y Lurena, 6 yugadas, y otras 4 que fueron de Antón de Faras, y otras 3 que fueron de Per Ibáñez de la Calzada. 
(5) Les concede en Benagra, "que es de suso de Benaborra" lo que fue de Esteban de Ferrera y de Gutierre Pérz (unas 12 yugadas) y «de suso de Benagira», 6 yugadas que fueron del hijo de Pedro Garcia Barba.

(6) Por último, les da en Cortigena 12 yugadas de tierra de labor que fueron de los hijos de Martín Meléndez.

(7) Todos estos heredamientos los concede el rey «con sus entrada $e$ con sus salidas, con montes e con pastos e con fuentes, e con todos sus términos, asi como siempre los hubieron en tiempo de moros".

(8) A cambio de estos heredamientos y por las franquicias y exenciones que el rey les hace, los pobladores estarán obligados a «velar cada año el castillo de Alcalá de Guadaira, e de no minguar vela ninguna de aquellos lugares acostumbrados de velar».

(9) Además, deberian poner «montaraces de cada año, aquellos que entendiere el nuestro alcade que estuviere y por nos, que cumplan el atalaya de cada día en el castillo, e demás velar el Arrabal, según entendiere lque cumplel, e lo velen por siempre jamás en tal manera que moredes en el castillo con vuestros cuerpos".

(10) «Que non podades vender ni empeñar ni enagenar estos heredamientos que nos vos damos / si no seis años adelante de la era en que fue fecha esta carta /; e los seis años adelante, que lo podades / vender, empeñar o enagenar a quien vos quisiéredes", siempre que sea hombre que «quiera cumplir esto que vos / avedes a / cumplir»; y no sea hombre de Orden o religión.

(11) Si alguno de los pobladores muriese y dejase como heredero a un «fijo pequeño... que non sea de edad e non oviese quien lo cumplir, los otros que fincaredes vivos que vos paredes a servir por él fasta que los herederos sean de edad para servir, con los esquilmos de los heredamientos".

(12) «Otrosi, si por aventura si alguno fasta los seis años lo cautivasen que pueda vender este heredamiento para salir de cautivo a quienquier que faga vecindad en Alcalá e cumpla / el servicio / que él habia a cumplir».

(13) «Et otrosí, por vos más bien e más merced, por este servicio que nos avedes a facer, que hayais la franqueza que han los vecinos de Sevilla, e que seais quitos e franqueados de todo pecho e de todo pedido e de préstamo, e de huéspedes e de toda facendera», y que hagan "vecindad con los vecinos de Sevilla, asi como los otros castillos de su término". 


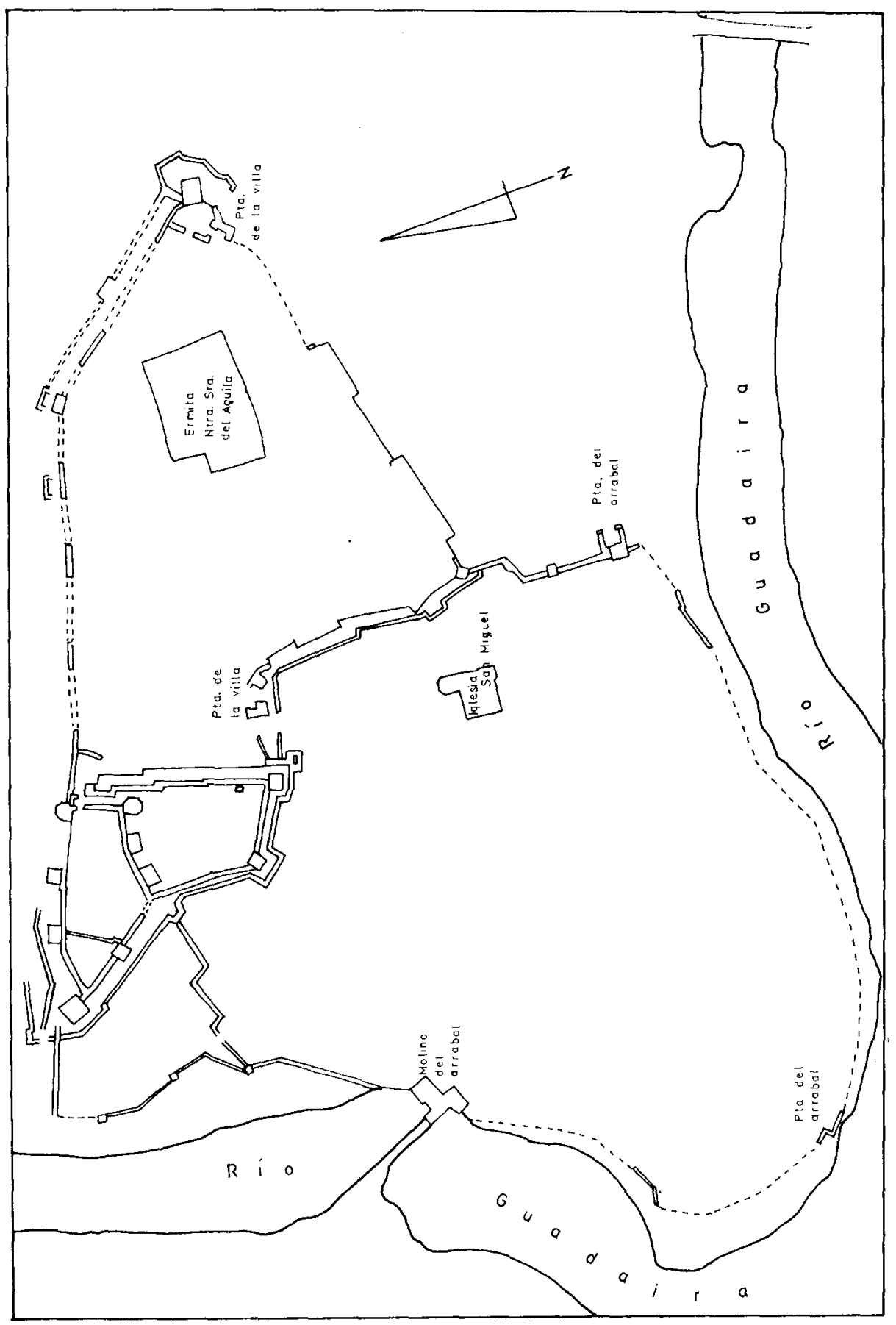




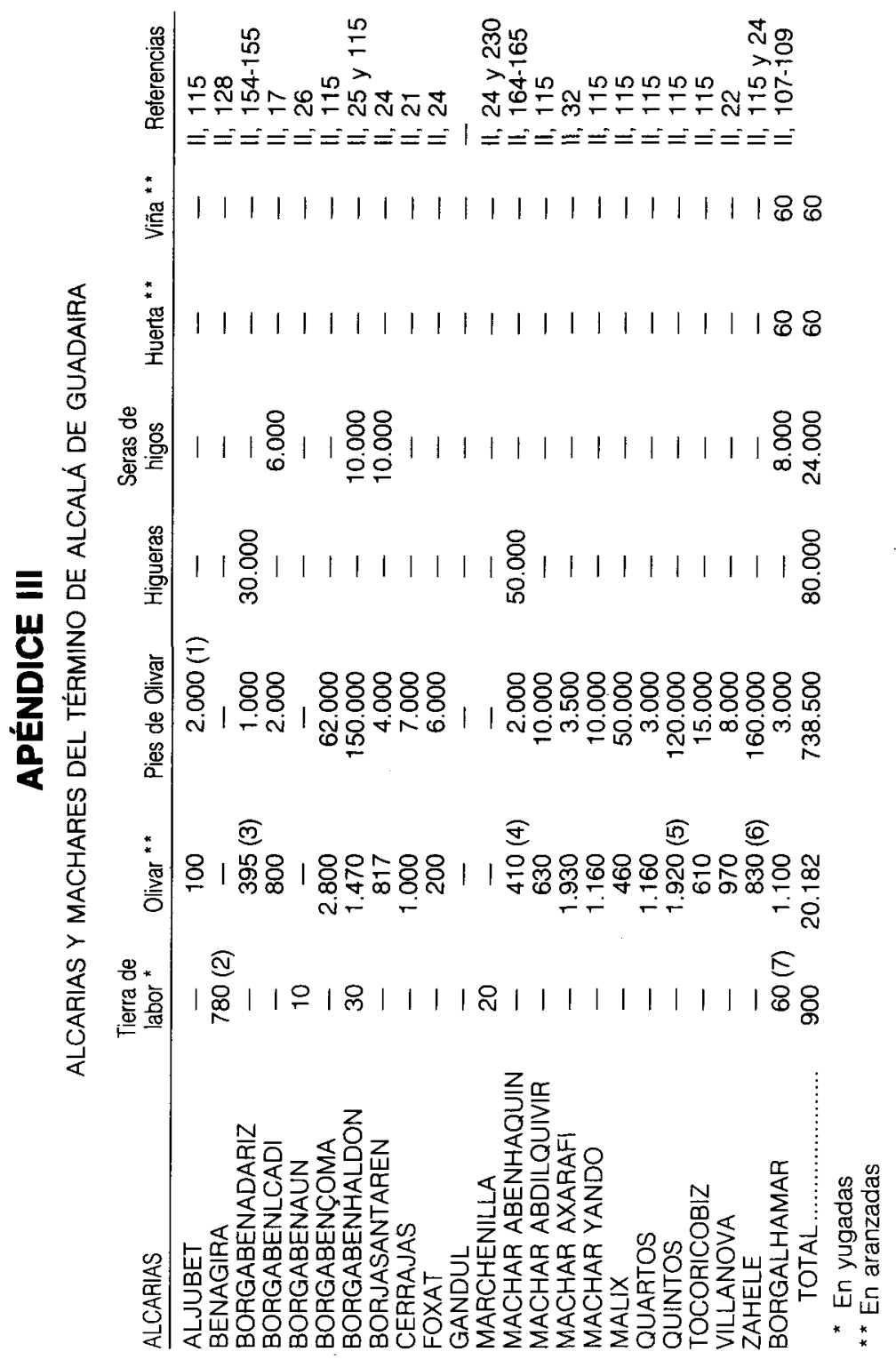

NOTAS:

(1) Olivar e higueral. Todas las referencias aluden al vol. II del Repartimiento de Sevilla, ed. por J. GONZÁLEZ

(2) Se incluyen las tierras dadas en CORTIGENA, LA MEMBRILLA y otros lugares sin identiticar. II, 207-225.

(3) Se computan 175 ars. dadas por el rey a diversas personas.

(4) Sólo se repartieron 337 ars.

(5) Se incluyen 100 ars. dadas a Guillén de Bec o Veque.

(6) Rodrigo Álvarez poseia la mitad de la alcaria, II, 115, O, según otro pasaje (II, 24), 300 ars.

(7) No se indica con precisión la ubicación de las parcelas de tierra calma, viña y huerta. 